\title{
Zero-dimensional polariton laser in a subwavelength grating-based vertical microcavity
}

\author{
Bo Zhang ${ }^{1}$, Zhaorong Wang ${ }^{1}$, Sebastian Brodbeck ${ }^{2}$, Christian Schneider ${ }^{2}$, Martin Kamp ${ }^{2}$, Sven Höfling ${ }^{2,3}$ \\ and Hui Deng ${ }^{1}$
}

Semiconductor exciton-polaritons in planar microcavities form coherent two-dimensional condensates in non-equilibrium. However, the coupling of multiple lower-dimensional polariton quantum systems, which are critical for polaritonic quantum device applications and novel cavity-lattice physics, has been limited in conventional cavity structures. Here, we demonstrate full non-destructive confinement of polaritons using a hybrid cavity composed of a single-layer subwavelength grating mirror and a distributed Bragg reflector. Single-mode polariton lasing was observed at a chosen polarization. The incorporation of a designable slab mirror in a conventional vertical cavity, when operating in the strong-coupling regime, enables the confinement, control and coupling of polariton gasses in a scalable fashion. This approach may open the door to experimental implementations of polariton-based quantum photonic devices and coupled cavity quantum electrodynamic systems.

Light: Science \& Applications (2014) 3, e135; doi:10.1038/Isa.2014.16; published online 3 January 2014

Keywords: Bose-Einstein condensation; microcavity; photonic crystal; polariton

\section{INTRODUCTION}

Semiconductor microcavity exciton-polaritons ${ }^{1}$ have recently emerged as a unique, open system for studying non-equilibrium quantum order. ${ }^{2-4}$ Exciton-polaritons are formed via strong coupling between excitons and photons. Due to the excitonic component, the polaritons are massive, weakly interacting quasiparticles that feature strong nonlinearity and rich many-body physics. ${ }^{5}$ By mixing with the photon, polaritons have an effective mass that is $10^{-8}$ of the hydrogen atomic mass, and they are relatively insensitive to disorder or localization potentials in the active media. Hence, polaritons exhibit quantum coherence over macroscopic scales with high critical temperatures. Polaritons in quantum-well microcavities couple out of the cavity at a fixed rate while conserving the energy and in-plane wavenumber, providing direct experimental access that is unavailable in typical many-body quantum systems. Hallmarks of non-equilibrium condensation and superfluidity have been widely observed in isolated two-dimensional (2D) polariton systems (Ref. 3 and the references therein).

Foundational work on 2D polariton systems has inspired theoretical schemes for polariton-based quantum circuits, ${ }^{6-8}$ quantum light sources $^{9-12}$ and novel quantum phases. ${ }^{4}$ Experimental implementation of these schemes requires the control, confinement and coupling of polariton systems, which remain challenging in conventional microcavity structures. Important features of a versatile experimental platform based on polaritons include: first, well-defined zero-dimensional (0D) polaritons as building blocks of a coupled system; second, the establishment (i.e., survival) of a non-equilibrium quantum phase in each $0 \mathrm{D}$ polariton cell, typically manifested as polariton lasing; third, controllable coupling among the 0D cells; fourth, individual addressability and control of each cell.

In conventional polariton cavities, the thick mirrors, consisting of distributed Bragg reflectors (DBRs), make it difficult for the polaritons to be confined or controlled beyond the perturbative regime. Most existing methods for controlling polaritons lead to a weak modulation potential that modifies the system's properties without reducing its dimensionality from 2D to 0D. Examples include weak confinement of excitons via mechanical strain ${ }^{13}$ and periodic modulation of the optical modes via surface patterning. ${ }^{14,15}$ Advanced techniques have been developed to embed apertures inside the cavity, ${ }^{16,17}$ which have led to $0 \mathrm{D}$ polariton cells, but polariton lasing has not been reported thus far. Alternatively, 0D polariton systems have also been produced via direct etching of the vertical cavity into pillars. ${ }^{18-21}$

Using this method, two groups have recently achieved polariton lasing in pillars, ${ }^{2-24}$ thus satisfying the first two requirements. However, this approach requires destructive plasma etching throughout the 4- to 6$\mu \mathrm{m}$-tall cavity structure as well as the active media layers, which preludes coupling between separate pillars. It is also unclear whether further control of the polariton modes in each pillar would be possible.

In this work, we demonstrate a polariton system in an unconventional cavity that can fulfill all four requirements. The new cavity structure replaces the top DBR with a slab of photonic crystal (PC) (Figure 1), which enables confinement and control of the polariton modes by

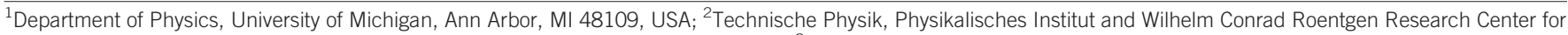

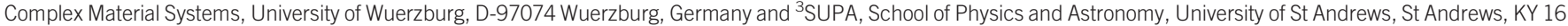
9SS, United Kingdom 


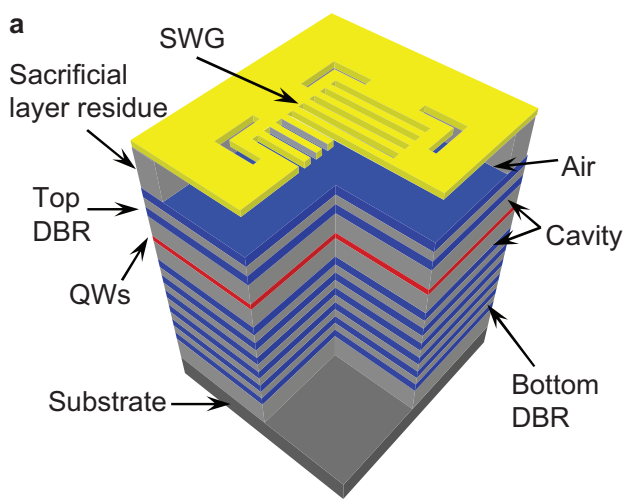

b

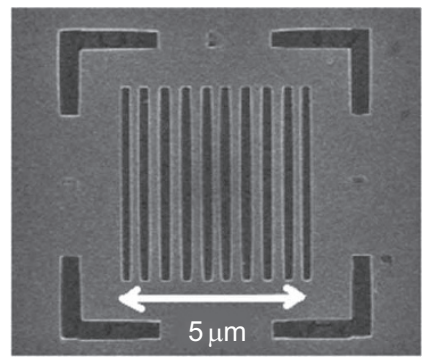

Figure 1 Examples of the hybrid cavity. (a) A schematic of a OD hybrid cavity with a SWG mirror. (b) Top-view SEM image of a fabricated OD cavity with a SWG of $5 \mu \mathrm{m} \times 5 \mu \mathrm{m}$ in size. OD, zero-dimensional; SEM, scanning electron microscopy; SWG, subwavelength grating.

design. $^{25-27}$ At the same time, there is no destructive interface in the active media layers or the main cavity layers; hence, coupling among multiple low-dimensional polariton cells is unhindered. Using this cavity system, we demonstrate $0 \mathrm{D}$ polariton lasing at a chosen polarization.

\section{MATERIALS AND METHODS}

A schematic of our hybrid cavity polariton device is shown in Figure 1a. To fabricate the device, we first grew the planar structure by molecular beam epitaxy on a GaAs substrate, consisting of 30 pairs of bottom DBR, an AlAs $\lambda / 2$ cavity layer, 2.5 pairs of top DBR consisting of $\mathrm{Al}_{0.15} \mathrm{GaAs} / \mathrm{AlAs}$, and an $\mathrm{Al}_{0.85} \mathrm{GaAs}$ sacrificial layer followed by an $\mathrm{Al}_{0.15} \mathrm{GaAs}$ top layer. There are $12 \mathrm{GaAs}$ quantum wells distributed in the three central antinodes of the cavity. We created square gratings of $5-8 \mu \mathrm{m}$ in length (Figure $1 \mathrm{~b}$ ) on the top layer via electron-beam lithography followed by a short plasma etching step. Hydrochloric acid chemical etching was then applied to remove the sacrificial layer, followed by critical point drying. The fabricated gratings are approximately $80 \mathrm{~nm}$ thick, with a period of approximately $520 \mathrm{~nm}$ and a duty cycle of approximately $40 \%$, and are suspended over an air gap of approximately $300 \mathrm{~nm}$. The gratings are optimized as a high-reflectance mirror for light polarized along the grating bar direction (transverse electric (TE)polarization). Figure $1 \mathrm{~b}$ shows a scanning electron microscopy image of the top view of one of the devices.

Optical measurements were performed to characterize the properties of the cavity system. For consistency, all data shown were taken on a device of $7.5 \mu \mathrm{m} \times 7.5 \mu \mathrm{m}$ in size. The sample was kept at $10-90 \mathrm{~K}$ in a continuous flow liquid-helium cryostat. A pulsed Ti:sapphire laser
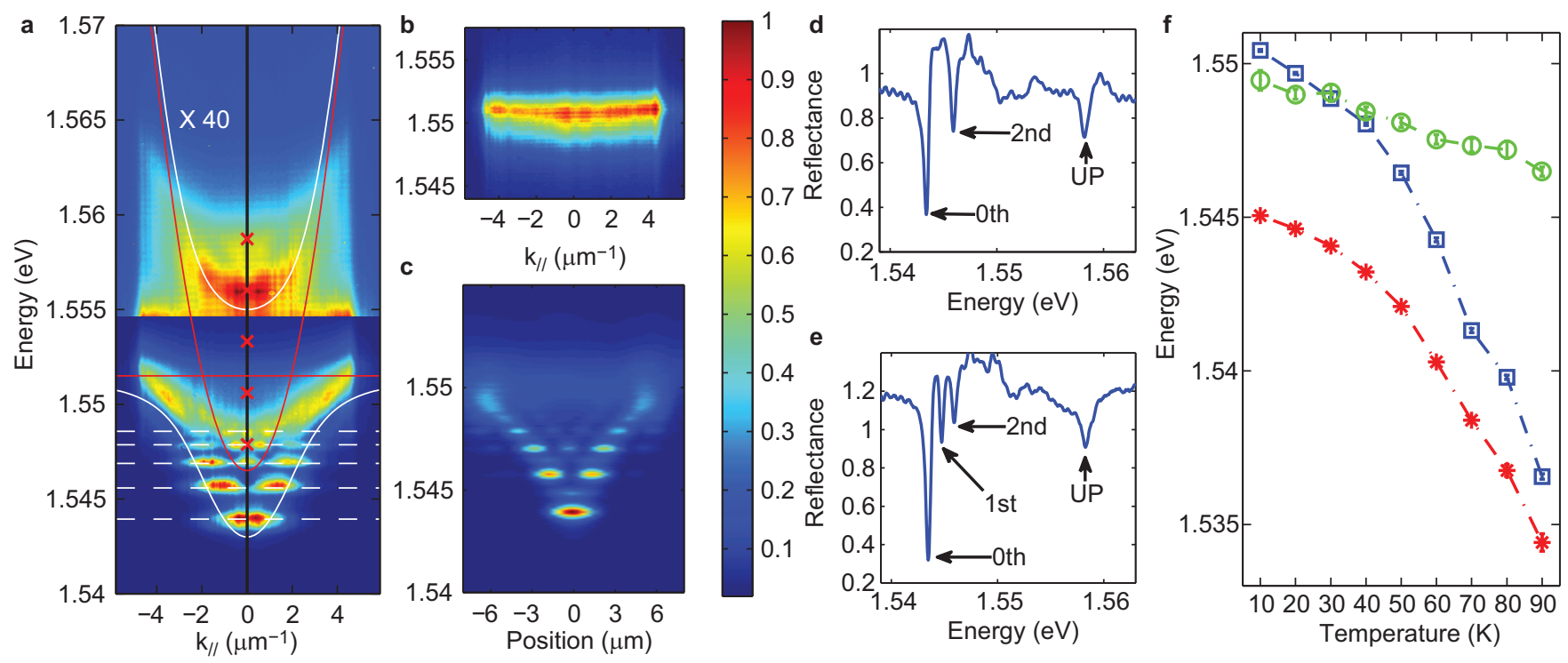

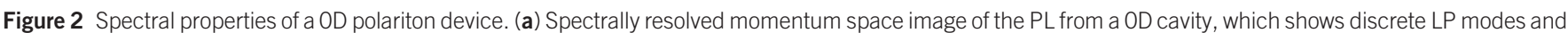

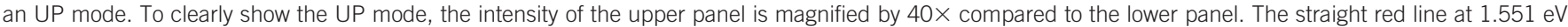

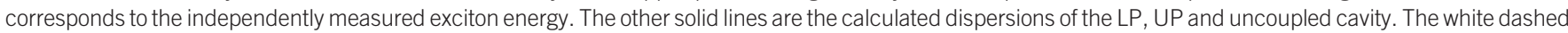

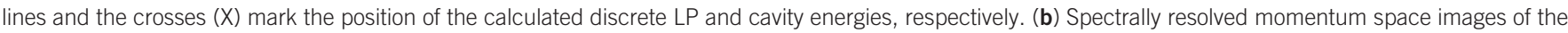

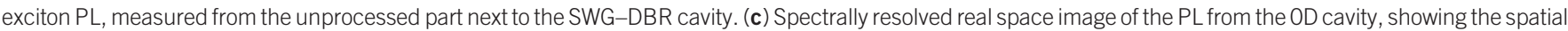

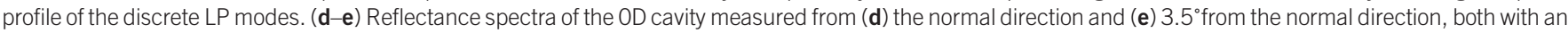

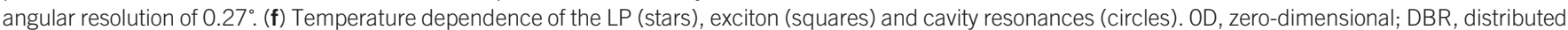
Bragg reflector; LP, lower polariton; PL, photoluminescence; SWG, subwavelength grating; UP, upper polariton. 
at $740 \mathrm{~nm}$ was used as the excitation laser, with an $80-\mathrm{MHz}$ repetition rate and a 100-fs pulse duration. The laser was focused to a spot size of approximately $2 \mu \mathrm{m}$ in diameter on the device from the normal direction using an objective lens with a numerical aperture of 0.55 . The photoluminescence signal was collected with the same objective lens, followed by real space or Fourier space imaging optics, and then sent to a $0.5-\mathrm{m}$ spectrometer with an attached nitrogen-cooled chargecoupled device. The spectrally resolved real space and Fourier space distributions were measured by selecting a strip across the center of the Fourier space and real space distributions using the spectrometer's entrance slit. The resolution of the measurements was limited by the charge-coupled device pixel size to $0.3 \mu^{-1}$ for Fourier space imaging and by the diffraction limit to $0.4 \mu \mathrm{m}$ for real space imaging.

\section{RESULTS AND DISCUSSION}

Strong coupling between the excitons and TE cavity modes was evident in the momentum space images of the emission from within the cavity, as shown in Figure 2a. Discrete lower polariton (LP) modes and a faint upper polariton (UP) branch were observed below and above the exciton energy, respectively, with dispersions distinct from that of the cavity photon (the red solid line). In contrast, the emission from outside the hybrid cavity region shows a flat, broad exciton band at the heavy hole exciton energy of $E_{\text {exc }}=1.551 \mathrm{eV}$ (Figure $2 \mathrm{~b}$ ). The energies of the polariton modes can be described as follows in the rotating wave approximation:

$$
\begin{aligned}
& E_{U P, L P}(k)= \\
& 1 / 2\left[E_{\text {exc }}(k)+E_{\text {cav }}(k) \pm \sqrt{\left(E_{\text {exc }}(k)-E_{\text {cav }}(k)\right)^{2}+4 \hbar^{2} \Omega^{2}}\right]
\end{aligned}
$$

Here, $k$ is the in-plane wavenumber, $E_{\text {cav }}$ is the uncoupled cavity energy and $2 \hbar \Omega$ is the exciton-photon coupling strength, corresponding to LP-UP splitting at zero exciton-photon detuning. Using Equation (1) and the measured $E_{\text {exc }}(k=0)=1.551 \mathrm{eV}, E_{L P}(k=0)=1.543 \mathrm{eV}$ and $E_{U P}(k=0)=1.556 \mathrm{eV}$, we obtain $E_{c a v}(0)=1.548 \mathrm{eV}$ and $2 \hbar \Omega=12 \mathrm{meV}$.

The discrete LP modes show full three-dimensional confinement of the polaritons. The lateral size of the hybrid cavity is determined by the size of the high-reflectance subwavelength grating (SWG). Outside the SWG, there is no cavity resonance, and the excitons are eigen-excitations. Inside the SWG region, the TE-polarized cavity modes strongly couple to the excitons, leading to laterally confined TE-polarized polariton modes. The transverse magnetic (TM)-polarized excitons remain in the weak coupling regime. Because there is not a sharp lateral boundary at which the cavity mode disappears, we phenomenologically modeled the effective confinement potential as an infinite harmonic potential. The calculated energies of the LP modes are indicated by the dashed lines in Figure 2a, which agree very well with the measured LP resonances. For comparison, the confined cavity modes (crosses) and corresponding 2D dispersions of the LP, UP and cavity modes are also shown (solid lines).

The spatial profiles of the confined LP modes were also measured via spectrally resolved real space imaging, as shown in Figure 2c. The four lowest LP modes are well confined within the SWG region, while the higher excited states are spread outside and form a continuous band. The variances of the $k$-space and $x$-space wavefunctions along the detected direction are $\Delta k=0.85 \mu \mathrm{m}$ and $\Delta x=1.01 \mu \mathrm{m}$. Their product is $\Delta x \times \Delta k=0.86$, slightly larger than the uncertainty limit of 0.5 , which may be due to the diffusion of the LPs.

The absorption spectra of the modes were obtained via reflectance measurements. The spectrum measured normal to the sample (Figure 2d) shows the three symmetric modes with the lowest mean in-plane wavenumber: the UP ground state, the LP ground state and
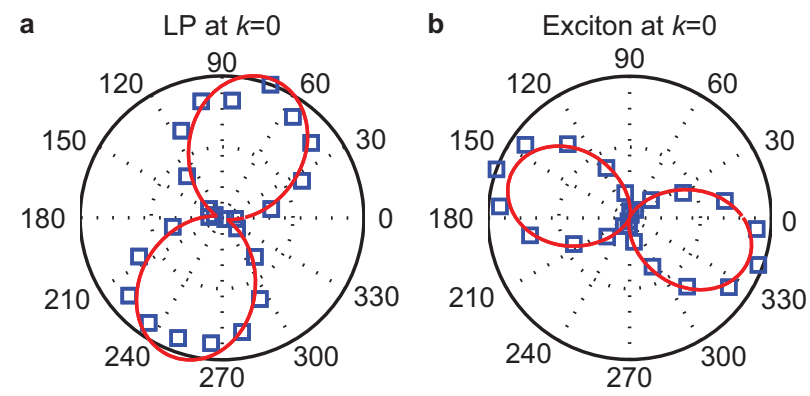

Figure 3 Polarizations of the polaritons and excitons in the hybrid-cavity polariton system. (a) Polar plots of the LP ground state intensity as a function of the angle of the linear polarization analyzer. The symbols represent the data. The solid lines fit to Equation (3), with a corresponding fitted linear degree of polarization of $91.9 \%$. (b) Polar plot for the exciton emission intensity from within the SWG, corresponding to a fitted linear degree of polarization of $98.2 \%$, with orthogonal polarization compared to (a). LP, lower polariton; SWG, subwavelength grating.

the second LP excited states. The spectral weights of the other polariton states are too small to be measured in reflectance. When measured at $3.5^{\circ}$ from the sample normal, the first excited state of the LPs was also observed (Figure 2e).

A further confirmation of the strong-coupling regime is the temperature tuning of the resonances, as shown in Figure 2f. As the temperature increased, the LP and UP ground state energies were redshifted and were measured via $k$-space photoluminescence. The exciton energy was directly measured in the planar region outside the SWG. The shift of the cavity photon energy was obtained from the shift of the first low-energy side minimum of the stopband. Anticrossing of the LP and UP modes is evident. From the LP, exciton and cavity energies, we obtain a coupling strength of $2 \hbar \Omega(T) \sim 10 \mathrm{meV}$ from $10 \mathrm{~K}$ to $80 \mathrm{~K}$, showing that strong coupling persists to the liquid nitrogen temperature and higher.

Unlike planar DBRs, the grating breaks the in-plane rotational symmetry. As a result, the SWG mirrors can have high polarization selectivity. We optimized our SWG to have high reflectance for the TE mode and low reflectance for the orthogonal TM mode. Correspondingly, the polaritons are TE-polarized, while the TMpolarized excitons remain in the weak coupling regime. Figure 3 shows the photoluminescence intensity $v s$. the angle of linear polarization for the LPs and excitons at $k \sim 0$ within the SWG region, normalized by the maximum intensity. We fit the data with $I=A \cos (\theta-\phi)^{2}+B$, where the fitting parameter $\phi$ depends on the orientation of the device, $A$ corresponds to linearly polarized light, and $B$ corresponds to a nonpolarized background. Correspondingly, the degree of linear polarization is $\mathrm{DOP}=\left(I_{\max }-I_{\min }\right) /\left(I_{\max }+I_{\min }\right)=A /(A+2 B)$. We obtained $A_{L P}=1.04 \pm 0.04, B_{L P}=0.05 \pm 0.01, \varphi_{L P}=71^{\circ} \pm 1^{\circ}$ and $\mathrm{DOP}=91.9 \%$ for the LPs, confirming that the LPs are highly TE-polarized. For the excitons, we obtained $A_{\text {exc }}=0.891 \pm 0.001, \quad B_{\text {exc }}=0.0081 \pm 0.0002$, $\varphi_{\text {exc }}=161^{\circ} \pm 1^{\circ}=\varphi_{L P}+90^{\circ}$ and DOP $=98.2 \%$, showing that the excitons are polarized orthogonal to the LPs. Such control of the polariton polarization has not been possible with conventional DBR-DBR cavities and is unique to the SWG-based cavity.

Finally, we show that polariton lasing was achieved in the 0D hybrid cavity. As shown in Figure 4a, the emission intensity I from the LP ground state increases sharply with the excitation power $P$ at a threshold of $P_{t h}=\sim 5 \mathrm{~kW} \mathrm{~cm}{ }^{-2}$, characteristic of the onset of lasing. Interestingly, the emission intensity $I$ varies with $P$ quadratically both below and well above the threshold, except at very low excitation densities. This result may arise because the energy separation between the discrete modes is larger 

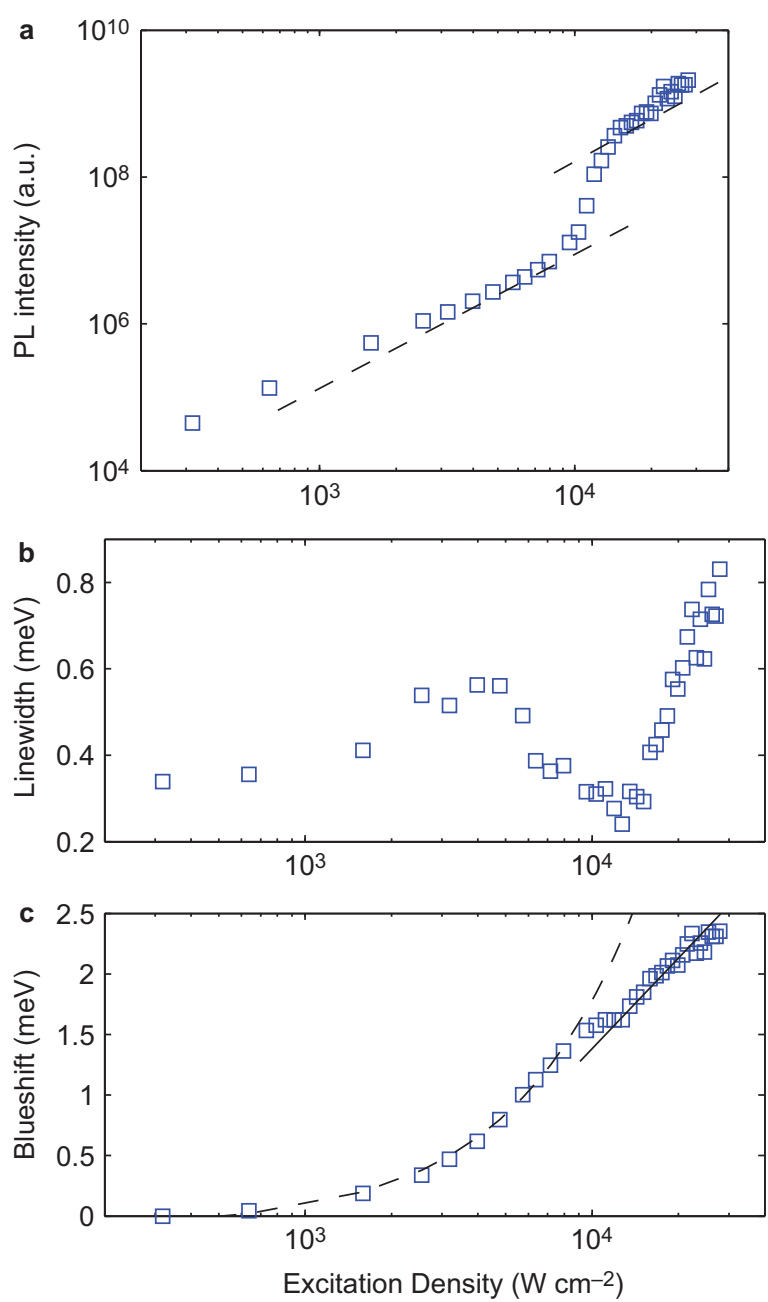

Figure 4 Lasing properties of the OD polaritons. (a) Integrated intensity, (b) linewidth and (c) corresponding energy blueshift of the LP ground state vs. the excitation density. The dashed lines in (a) provide a comparison with quadratic dependence. The dashed lines in (c) display comparisons with the linear dependence below the threshold and logarithmic dependence above the threshold. OD, zero-dimensional; LP, lower polariton; PL, photoluminescence.

than $k_{B} T \sim 0.8 \mathrm{meV}$. As a result, relaxation to the ground state through LPphonon scattering is suppressed compared to LP-LP scattering.

Accompanying the transition, a sharp decrease in the LP ground state linewidth was measured. The minimum linewidth of $0.24 \mathrm{meV}$ may be primarily limited by the intensity fluctuation of the pulsed excitation laser. ${ }^{28}$ The LP energy increased continuously with the excitation density due to exciton-exciton interactions. The blueshift shows a linear dependence below the threshold, is suppressed near the threshold, and shows a logarithmic dependence above the threshold. ${ }^{22,29}$ The discrete energy levels are maintained across the threshold and remain distinct below the uncoupled cavity energy.

The establishment of polariton lasing confirms the quality of the $0 \mathrm{D}$ polariton system. The threshold density is smaller than or comparable to those measured in DBR-DBR pillar cavities. ${ }^{22,23}$ The linewidth reduction and blueshift are all within an order of magnitude of reported values in DBR-DBR planar or pillar microcavities. ${ }^{1,22,23}$ Unlike DBR-DBR cavities, however, the polariton lasing demonstrated herein occurs with a priori defined polarization, independent of the excitation conditions.

\section{CONCLUSIONS}

In conclusion, we have demonstrated the first hybrid cavity incorporating a slab PC mirror, operating in the strong coupling regime and supporting polariton lasing. Three-dimensional confinement of the polaritons was achieved by using a finite size SWG, with the quantum wells and the main cavity layers untouched. Polariton lasing in the ground state was readily observed.

Unique to the hybrid SWG cavity, the LP is linearly polarized, while the orthogonally polarized exciton mode remains in the weak-coupling regime. The PL of the weakly coupled TM excitons provides direct access to the TE exciton reservoir, which has not been available in conventional III-As cavities. This approach can enable polarized polariton lasers ${ }^{30-34}$ and can simplify quantum photonic devices based on single-spin polaritons. ${ }^{10-12,35}$

The integration of a slab PC mirror in a polariton system adds flexibility in controlling the fundamental properties of polaritons by design, including the dimensionality and polarization, as demonstrated in this work. The investigation of different PC designs will allow for further modifications of the polariton properties. Importantly, control of the polariton system was achieved without creating destructive interfaces in the active media or main cavity layer. Hence, extension of this single $0 \mathrm{D}$ polariton system to multiple close-placed systems would allow for the creation of controllably coupled polariton systems, while each $0 \mathrm{D}$ cell in the coupled system could be separately controlled and probed. The demonstrated hybrid-cavity polariton system may provide a scalable architecture for the experimental implementation of coupled lattice cavity systems. ${ }^{4,36}$

\section{ACKNOWLEDGMENTS}

BZ, ZW and HD acknowledge support from the National Science Foundation under Awards DMR 1150593 for measurements and OISE 1132725 for travel expenses and the Air Force Office of Scientific Research under Award FA955012-1-0256 for device fabrication and characterization. CS, SB, MK and SH acknowledge support from the State of Bavaria, Germany. Fabrication of the SWG was performed at the Lurie Nanofabrication Facility, which is part of the National Science Foundation NNIN network.

1 Weisbuch C, Nishioka M, Ishikawa A, Arakawa Y. Observation of the coupled excitonphoton mode splitting in a semiconductor quantum microcavity. Phys Rev Lett 1992; 69: 3314-3317.

2 Keeling J, Berloff NG. Condensed-matter physics: going with the flow. Nature 2009, 457: 273-274.

3 Deng H, Haug H, Yamamoto Y. Exciton-polariton Bose-Einstein condensation. Rev Mod Phys 2010; 82: 1489-1537.

4 Carusotto I, Ciuti C. Quantum fluids of light. Rev Mod Phys 2013; 85: 299-366.

5 Littlewood PB, Eastham PR, Keeling JM, Marchetti FM, Simon BD et al. Models of coherent exciton condensation. J Phys Cond Matter 2004; 16: S3597-S3620.

6 Liew TCH, Kavokin AV, Shelykh IA. Optical circuits based on polariton neurons in semiconductor microcavities. Phys Rev Lett 2008; 101: 016402.

7 Shelykh IA, Johne R, Solnyshkov DD, Malpuech G. Optically and electrically controlled polariton spin transistor. Phys Rev B 2010; 82: 153303.

8 Liew TC, Kavokin AV, Ostatnick T, Kaliteevski M, Shelykh IA et al. Excitonpolariton integrated circuits. Phys Rev B 2010; 82: 033302.

9 Verger A, Ciuti C, Carusotto I. Polariton quantum blockade in a photonic dot. Phys Rev B 2006; 73: 193306.

10 Liew TC, Savona V. Quantum entanglement in nanocavity arrays. Phys Rev A 2012; 85: 050301.

11 Majumdar A, Bajcsy M, Rundquist A, Vuckovic J. Loss-enabled sub-Poissonian light generation in a bimodal nanocavity. Phys Rev Lett 2012; 108: 183601.

12 Liew TC, Savona V. Multimode entanglement in coupled cavity arrays. New J Phys 2013; 15: 025015

13 Balili R, Hartwell V, Snoke D, Pfeiffer L, West K. Bose-Einstein condensation of microcavity polaritons in a trap. Science 2007; 316: 1007-1010.

14 de Lima MM, van der Poel M, Santos PV, Hvam JM. Phonon-induced polariton superlattices. Phys Rev Lett 2006; 97: 045501.

15 Lai CW, Kim NY, Utsunomiya S, Roumpos G, Deng H et al. Coherent zero-state and $\pi$-state in an excitonpolariton condensate array. Nature 2007; 450: 529-532. 
16 Daif OE, Baas A, Guillet T, Brantut JP, Kaitouni RI et al. Polariton quantum boxes in semiconductor microcavities. Appl Phys Lett 2006; 88: 061105.

17 Winkler K, Schneider C, Fischer J, Rahimi-Iman A, Amthor M et al. Electroluminescence from spatially confined exciton polaritons in a textured microcavity. Appl Phys Lett 2013; 102: 041101.

18 Tartakovskii A, Kulakovskii V, Koval Y, Borzenko T, Forchel A et al. Excitonphoton interaction in low-dimensional semiconductor microcavities. J Exp Theor Phys 1998; 87: 723-730.

19 Gutbrod T, Bayer M, Forchel A, Reithmaier JP, Reinecke TL et al. Weak and strong coupling of photons and excitons in photonic dots. Phys Rev B 1998; 57: 9950-9956.

20 Dasbach G, Bayer M, Schwab M, Forchel A. Spatial photon trapping: tailoring the optical properties of semiconductor microcavities. Semicond Sci Technol 2003; 18: S339.

21 Dasbach G, Diederichs C, Tignon J, Ciuti C, Roussignol P et al. Polarization inversion via parametric scattering in quasi-one-dimensional microcavities. Phys Rev B 2005; 71: 161308 .

22 Bajoni D, Senellart P, Wertz E, Sagnes I, Miard A et al. Polariton laser using single micropillar GaAs-GaAlAs semiconductor cavities. Phys Rev Lett 2008; 100: 047401.

23 Brichkin AS, Novikov SI, Larionov AV, Kulakovskii VD, Glazov MM et al. Effect of Coulomb interaction on exciton-polariton condensates in GaAs pillar microcavities. Phys Rev B 2011; 84: 195301.

24 Kulakovskii VD, Brichkin AS, Novikov SV, Schneider C, Höfling S et al. Magnetic field control of polarized polariton condensates in rectangular microcavity pillars. Phys Rev B 2012; 85: 155322.

25 Mateus CF, Huang MC, Deng YF, Neureuther AR, Chang-Hasnain CJ. Ultrabroadband mirror using low-index cladded subwavelength grating. IEEE Photon Technol Lett 2004; 16: 518-520.

26 Jung IW, Kim S, Solgaard O. High-Reflectivity broadband photonic crystal mirror MEMS scanner with low dependence on incident angle and polarization. $J$ Microelectromech Syst 2009; 18: 924-932.
27 Fattal D, Li J, Peng Z, Fiorentino M, Beausoleil RG. Flat dielectric grating reflectors with focusing abilities. Nat Photon 2010; 4: 466-470.

28 Love AP, Krizhanovskii DN, Whittaker DM, Bouchekioua R, Sanvitto D et al. Intrinsic decoherence mechanisms in the microcavity polariton condensate. Phys Rev Lett 2008; 101: 067404.

29 Roumpos G, Nitsche WH, Höfling S, Forchel A, Yamamoto Y. Gain-induced trapping of microcavity exciton polariton condensates. Phys Rev Lett 2010; 104: 126403.

30 Deng H, Weihs G, Snoke D, Bloch J, Yamamoto Y. Polariton lasing vs. photon lasing in a semiconductor microcavity. Proc Natl Acad Sci USA 2003; 100: 15318-15323.

31 Christopoulos S, von Hgersthal GB, Grundy AJ, Lagoudakis PG, Kavokin AV et al. Room-temperature polariton lasing in semiconductor microcavities. Phys Rev Lett 2007; 98: 126405.

32 Kena-Cohen S, Forrest SR. Room-temperature polariton lasing in an organic singlecrystal microcavity. Nat Photon 2010; 4: 371-375

33 Das A, Heo J, Jankowski M, Guo W, Zhang L et al. Room temperature ultralow threshold GaN nanowire polariton laser. Phys Rev Lett 2011; 107: 066405.

34 Lu TC, Lai YY, Lan YP, Huang SW, Chen JR et al. Room temperature polariton lasing vs. photon lasing in a ZnO-based hybrid microcavity. Opt Express 2012; 20: 5530 5537

35 Liew TC, Savona V. Single photons from coupled quantum modes. Phys Rev Lett 2010; 104: 183601.

36 Hartmann M, Brando F, Plenio M. Quantum many-body phenomena in coupled cavity arrays. Laser Photon Rev 2008; 2: 527-556.

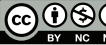

This work is licensed under a Creative Commons Attribution-

NonCommercial-NoDerivs Works 3.0 Unported license. To view a copy of this license, visit http://creativecommons.org/licenses/by-nc-nd/3.0 